\title{
THE EXACT FINITE-DIFFERENCE SCHEME FOR VECTOR BOUNDARY-VALUE PROBLEMS WITH PIECE-WISE CONSTANT COEFFICIENTS
}

\author{
H. KALIS \\ Institute of Mathematics and University of Latvia, \\ Akadēmijas laukums 1, LV-1524 Rīga, Latvija \\ E-mail: kalis@lanet.lv
}

\begin{abstract}
We will consider the exact finite-difference scheme for solving the system of differential equations of second order with piece-wise constant coefficients. It is well-known, that the presence of large parameters at first order derivatives or small parameters at second order derivatives in the system of hydrodynamics and magnetohydrodynamics (MHD) equations (large Reynolds, Hartmann and others numbers) causes additional difficulties for the applications of general classical numerical methods. Thus, important to work out special methods of solution, the so-called uniform converging computational methods. This gives a basis for the development of special monotone finite vector-difference schemes with perturbation coefficient of function-matrix for solving the system of differential equations. Special finite-difference approximations are constructed for a steady-state boundary-value problem, systems of parabolic type partial differential equations, a system of two MHD equations, 2-D flows and MHD-flows equations in curvilinear orthogonal coordinates.
\end{abstract}

\section{1-D LINEAR SYSTEM}

We start with a simple example of one dimensional (1-D) linear system of $\mathrm{m}$ differential equations of second order:

$$
L \mathbf{u} \equiv \partial(\lambda \partial \mathbf{u} / \partial x) / \partial x-a \partial \mathbf{u} / \partial x=\mathbf{f}
$$

where $x \in(0, l), \lambda>0, a$ is the matrix, $\mathbf{u}, \mathbf{f}$ are vectors. Let the coefficients of $\lambda, a, \mathbf{f}$ are piece-wise continuous in the interval $(0, l)$ and the nonuniform grid contains the discontinuity points of coefficients. The vector-function $\mathbf{u}$ and the flux vector-function $\lambda \partial \mathbf{u} / \partial x$ are continuous . 
We assume that the boundary conditions can be written as

$$
\begin{array}{cl}
\nu_{0} \lambda_{1} \partial \mathbf{u} / \partial x-\alpha_{0} \mathbf{u}=-\alpha_{0} \Phi_{0}, & x=0 \\
\nu_{1} \lambda_{N} \partial \mathbf{u} / \partial x+\alpha_{N} \mathbf{u}=\alpha_{N} \Phi_{1}, & x=l,
\end{array}
$$

where $\nu_{0}=0$ or $\nu_{1}=0$ for the Dirichlet boundary conditons $\left(\mathbf{u}=\Phi_{0}\right.$ at $x=0$ or $\mathbf{u}=\Phi_{1}$ at $x=l$ ) ;

$\nu_{0}=1$ or $\nu_{1}=1$ for the Neumann boundary conditions $\left(\alpha_{0}=0\right.$ or $\left.\alpha_{N}=0\right)$ or general form of boundary conditions;

$\alpha_{0}>0, \alpha_{N}>0, \lambda_{1}, \lambda_{N}$ are the matrix, $\Phi_{0}, \Phi_{1}$ are the vectors of order $\mathrm{m}$.

\section{3-POINT EXACT VECTOR-DIFFERENCE EQUATIONS}

Approximation of the differential problem is based on the conservation law approach of the finite volumes method [4].

We consider the nonuniform grid with blocks centered at the grid points

$$
x_{j}, \quad j=\overline{0, N}, \quad\left(x_{0}=0, x_{N}=l\right) .
$$

We will refer to the endpoints of the interval about the point $x_{j}$ as $x_{j \pm 0.5}$. This interval $\left(x_{j-0.5}, x_{j+0.5}\right)$ is refered as the control volume associated with the grid point $x_{j}$ (the $\mathrm{j}$-th cell). To derive a difference equation associated with the grid point $x_{j}$ we integrate the self-adjoint form of vector differential equation (1)

$$
\partial\left(\lambda^{*} \partial \mathbf{u} / \partial x\right) \partial x=\mathbf{G}(x)
$$

in the intervals $\left(x_{j-0.5}, x_{j+0.5}\right)$ :

$$
\mathbf{W}_{j+0.5}-\mathbf{W}_{j-0.5}=\int_{x_{j-0.5}}^{x_{j}} \mathbf{G}_{j} d z+\int_{x_{j}}^{x_{j+0.5}} \mathbf{G}_{j+1} d z
$$

where

$$
\begin{gathered}
\mathbf{W}_{j \pm 0.5}=\left.\mathbf{W}\right|_{x=x_{j \pm 0.5}}, \quad x_{j \pm 0.5}=\left(x_{j}+x_{j \pm 1}\right) / 2, \quad h_{j}=x_{j}-x_{j-1}, \\
\mathbf{W}(x)=\lambda^{*} \partial \mathbf{u} / \partial x, \lambda^{*}=J \lambda, \mathbf{G}=J \mathbf{f}, J(x)=\exp \left(-\int_{x_{j}}^{x} \lambda^{-1} a d t\right) .
\end{gathered}
$$

This is the integral form of the conservation law for the interval $\left(x_{j-0.5}, x_{j+0.5}\right)$. In the classical formulation of the finite volumes method [4], it is assumed that the vector flux terms $\mathbf{W}_{j \pm 0.5}$ are approximated by the finite differences. Then, the corresponding difference scheme is not exact for given vector-functions $\mathbf{G}_{j}$ 
even in the case of piece-wise constant coefficients of $\lambda, a, f$. We have an opportunity to derive the exact difference scheme in this case. We integrate equation (4) from $x_{j-0.5}$ to $x \in\left(x_{j-1}, x_{j}\right)$ :

$$
\mathbf{W}-\mathbf{W}_{j-0.5}=\int_{x_{j-0.5}}^{x} \mathbf{G}_{j} d \xi .
$$

Dividing this expression by $\lambda^{*}$ and integrating from $x_{j-1}$ to $x_{j}$ yields

$$
\mathbf{u}_{j}-\mathbf{u}_{j-1}=\left(A_{j}^{-}\right)^{-1} \mathbf{W}_{j-0.5}+\mathbf{B}_{j}
$$

where

$$
\left(A_{j}^{-}\right)^{-1}=\int_{x_{j-1}}^{x_{j}}\left(\lambda^{*}\right)^{-1} d x, \quad \mathbf{B}_{j}=\int_{x_{j-1}}^{x_{j}}\left(\lambda^{*}\right)^{-1} d x \int_{x_{j-0.5}}^{x} \mathbf{G}_{j} d \xi,
$$

and $\mathbf{u}_{j}, \mathbf{u}_{j-1}$ represent the values of the vector-function $\mathbf{u}$ at $x_{j}, x_{j-1}$. Hence

$$
\mathbf{W}_{j-0.5}=A_{j}^{-}\left(\mathbf{u}_{j}-\mathbf{u}_{j-1}\right)-A_{j}^{-} \mathbf{B}_{j} .
$$

Similarly, the flux term $\mathbf{W}_{j+0.5}$ is given by

$$
\mathbf{W}_{j+0.5}=A_{j+1}^{+}\left(\mathbf{u}_{j+1}-\mathbf{u}_{j}\right)-A_{j+1}^{+} \mathbf{B}_{j+1} .
$$

In order to derive a 3-point exact vector-difference equations associated with the central grid point $x_{j}$ we want to apply vector-equation (5) in the form

$$
\hbar_{j} \Lambda \mathbf{u}_{j} \equiv A_{j+1}^{+}\left(\mathbf{u}_{j+1}-\mathbf{u}_{j}\right)-A_{j}^{-}\left(\mathbf{u}_{j}-\mathbf{u}_{j-1}\right)=\mathbf{R}_{j}, \quad j=\overline{1, N-1}
$$

where $\hbar_{j}=0.5\left(h_{j}+h_{j+1}\right)$, and

$$
\begin{aligned}
& \mathbf{R}_{j}=\int_{x_{j-1}}^{x_{j}}\left(1-A_{j}^{-} \int_{x}^{x_{j}}\left(\lambda^{*}\right)^{-1} d \xi\right) \mathbf{G}_{j} d x \\
& +\int_{x_{j}}^{x_{j+1}}\left(1-A_{j+1}^{+} \int_{x_{j}}^{x}\left(\lambda^{*}\right)^{-1} d \xi\right) \mathbf{G}_{j+1} d x
\end{aligned}
$$

If the values of the parameters $\lambda, a, \mathbf{f}$ of equation (1) are equal to $\lambda_{j-0.5}, a_{j-0.5}$, $\mathbf{f}_{j-0.5}$ and $\lambda_{j+0.5}, a_{j+0.5}, \mathbf{f}_{j+0.5}$, in the intervals $\left(x_{j-1}, x_{j}\right),\left(x_{j}, x_{j+1}\right)$ respectively, then

$$
\begin{aligned}
& A_{j}^{-}=\frac{\lambda_{j-0.5}}{h_{j}} g\left(-\beta_{j}\right), A_{j+1}^{+}=\frac{\lambda_{j+0.5}}{h_{j+1}} g\left(\beta_{j+1}\right), \\
& \mathbf{R}_{j}=h_{j+1} r\left(\beta_{j+1}\right) \mathbf{f}_{j+0.5}+h_{j} r\left(-\beta_{j}\right) \mathbf{f}_{j-0.5}
\end{aligned}
$$

where

$$
g(s)=s(\exp (s)-E)^{-1}, r(s)=s^{-1}(E-g(s))
$$


are the functions of the matrix $s=\lambda^{-1} a h, E$ is the identity matrix of order $m$. In the case of a uniform grid and constants coefficients $\lambda, a, \mathbf{f}$ we have $h_{j}=h_{j+1}=h, \beta_{j}=\beta_{j+1}, g( \pm s)=\gamma(s) \pm s / 2, r(s)+r(-s)=E$, where $\gamma(s)=0.5 s \operatorname{coth}(0.5 s)$. The matrix-functions $g, r, \gamma$ can be calculated on the spectrum of the matrix $s$ [5]. Since the matrix-function $g(s)$ associated with the matrix $s$ has nonnegative eigenvalues, we have $A_{j}^{-}>0, A_{j+1}^{+}>0$ and the corresponding vector-difference scheme is monotone. This scheme can be solved by vector-factorization method for tridiagonal systems. The presence of the matrix-functions $g(s), r(s)$ in the case of piece-wise constant elements of the matrix $a, \lambda$ and vectors $\mathbf{f}$ ensure the exact discrete approximation of corresponding 1 - D boundary value problem.

\section{2-POINT EXACT VECTOR-DIFFERENCE EQUATIONS}

We apply the integral form of the conservation law to the half interval $\left(x_{0}, x_{0.5}\right)$ for the approximation of the boundary condition $(2)\left(\nu_{0}=1\right)$ in the form

$$
\mathbf{W}_{0.5}-\mathbf{W}_{0}=\int_{x_{0}}^{x_{0.5}} \mathbf{G}_{1} d z
$$

where

$$
\mathbf{W}_{0}=\left.\mathbf{W}\right|_{x=x_{0}}=\alpha_{0}\left(\mathbf{u}_{0}-\Phi_{0}\right), \mathbf{W}_{0.5}=\left.\mathbf{W}\right|_{x=x_{0.5}} .
$$

By integrating the equation (4) from $x_{0.5}$ to $x \in\left(x_{0}, x_{1}\right)$ and from $x_{0}$ to $x_{1}$, we can easily derive the following 2-point exact vector-difference equation associated with the grid point $x_{0}=0$ :

$$
h_{1} \Lambda \mathbf{u}_{0} \equiv A_{1}^{+}\left(\mathbf{u}_{1}-\mathbf{u}_{0}\right)-\alpha_{0}\left(u_{0}-\Phi_{0}\right)=\mathbf{R}_{0},
$$

where $A_{1}^{+}=\lambda_{0.5} / h_{1} g\left(\beta_{1}\right), \quad \beta_{1}=\lambda_{0.5}^{-1} a_{0.5} h_{1}$,

$$
\mathbf{R}_{0}=\int_{x_{0}}^{x_{1}}\left(1-A_{1}^{+} \int_{x_{0}}^{x}\left(\lambda^{*}\right)^{-1} d \xi\right) \mathbf{G}_{1} d x
$$

and $\mathbf{u}_{0}, \mathbf{u}_{1}$ represent the values of the vector-function $\mathbf{u}$ at $x_{0}, x_{1}$.

Similarly for the boundary condition (3) we obtain the 2-point exact vectordifference equation associated with the grid point $x_{N}=l$

$$
h_{N} \Lambda \mathbf{u}_{N} \equiv-\alpha_{N}\left(\mathbf{u}_{N}-\Phi_{1}\right)-A_{N}^{-}\left(\mathbf{u}_{N}-\mathbf{u}_{N-1}\right)=\mathbf{R}_{N},
$$

where $\quad A_{N}^{-}=\lambda_{N-0.5} h_{N}^{-1} g\left(-\beta_{N}\right), \quad \beta_{N}=\lambda_{N-0.5}^{-1} a_{N-0.5} h_{N}$,

$$
\mathbf{R}_{N}=\int_{x_{N-1}}^{x_{N}}\left(1-A_{N}^{-} \int_{x}^{x_{N}}\left(\lambda^{*}\right)^{-1} d \xi\right) \mathbf{G}_{N} d x=h_{N} r\left(-\beta_{N}\right) \mathbf{f}_{N-0.5}
$$


and $\mathbf{u}_{N-1}$ represents the value of the vector-function $\mathbf{u}$ at $x_{N-1}$.

Therefore, the vector-difference scheme (6),(9),(10) is monotone $\left(\alpha_{0} \geq 0, \alpha_{N} \geq\right.$ $\left.0, A_{j}^{-}>0, A_{j+1}^{+}>0\right)$ and has the unique solution.

\section{1-D NONLINEAR SYSTEM AND 1-D TIME DEPENDING SYSTEM}

The system of $m$ nonlinear differential equations (1) with the coefficients $\lambda, a, \mathbf{f}$ depending on $x, \mathbf{u}$ can be approximated in the form (6),(7), where $\lambda_{j \pm 0.5}, a_{j \pm 0.5}, \mathbf{f}_{j \pm 0.5}$ denote the discrete approximation of $\lambda, a, \mathbf{f}$ in the correspondigs intervals $\left(x_{j-1}, x_{j}\right),\left(x_{j}, x_{j+1}\right)$. The truncation error of the vectordifference equations is locally $O\left(\max \left|h_{j+1}-h_{j}\right|\right)$. For the uniform grid we obtain the accuracy of second order. The vector-difference scheme generalizes the corresponding monotone difference scheme in the scalar case.

The nonlinear time-depending 1-D initial-boundary-value problem for parabolic type system of $m$ partial differential equations is in the form (1)-(3), where $\mathbf{u}=\mathbf{u}(x, t), \mathbf{f}=\partial \mathbf{u} / \partial t-\mathbf{f}^{*}$ and the parameters $\lambda, a, \mathbf{f}^{*}$ are functions depending on $x, t, \mathbf{u}$. The corresponding discretized version of vector-equations (1) for the monotone difference scheme is given in the form [6]

$$
\left(\mathbf{u}_{j}^{n+1}-\mathbf{u}_{j}^{n}\right) / \tau=\sigma \Lambda \mathbf{u}_{j}^{n+1}+(1-\sigma) \Lambda \mathbf{u}_{j}^{n}+\mathbf{f}_{j}^{*},
$$

where $\mathbf{u}_{j}^{n}, \mathbf{f}_{j}^{*}$ denote the discrete approximations of $\mathbf{u}, \mathbf{f}^{*}$ at the grid point $x_{j}$ and at the time $t=t_{n}=n \tau, n=0,1, \cdots, \tau$ is the time step, $\sigma \in(0,1)$ is the weight-parameter of schemes, $\Lambda \mathbf{u}_{j}$ are the vector difference expressions of (6),(9),(10); $j=\overline{1, N-1}$. If $\sigma \neq 0.5$ then the truncation error of (11) is locally of the first order in time, in case $\sigma=0.5$ it is of the second order.

The stability condition in the case of constant coefficients and uniform grid has the form

$$
\|\lambda\| k_{0}(1-\sigma) \max \left|\gamma_{j}\right| \leq 1
$$

where $\gamma_{j}=\left(\mu_{j} / 2\right) \operatorname{coth}\left(\mu_{j} / 2\right), \mu_{j}$ are the eigenvalues of the matrix $s, j=$ $\overline{1, m}, k_{0}=2 \tau / h^{2}$.

\section{2-D TIME DEPENDING SYSTEM}

We consider the nonlinear time depending 2-D initial-boundary-value problem for parabolic system of $m$ partial differential equations in the bounded domain $D=\left\{(x, y): x \in(0, l), y \in\left(0, l^{*}\right)\right\}$, has the form (1), where $\mathbf{u}=\mathbf{u}(x, y, t)$,

$$
\mathbf{f}=\partial \mathbf{u} / \partial t-\partial(\lambda \partial \mathbf{u} / \partial y) / \partial y+a^{*} \partial \mathbf{u} / \partial y-\mathbf{f}^{*},
$$

$\lambda>0, a, a^{*}, \mathbf{f}^{*}$ are matrix and vector functions depending on $x, y, t, \mathbf{u}$.

The corresponding difference equations (11) can be rewritten in the form [6]

$$
\left(\mathbf{u}_{j, i}^{n+1}-\mathbf{u}_{j, i}^{n}\right) / \tau=\sigma \Lambda \mathbf{u}_{j, i}^{n+1}+(1-\sigma) \Lambda \mathbf{u}_{j, i}^{n}+\mathbf{f}_{i, j}^{*},
$$


The exact finite-difference scheme for vector boundary-value problems 119

where $\mathbf{u}_{j, i}^{n}, \mathbf{f}_{j, i}^{*}$ denote the disrete approximation of $\mathbf{u}, \mathbf{f}^{*}$ at the grid point $\left(x_{j}, y_{i}\right)$ and at time $t=t_{n}=n \tau$,

$$
\Lambda \mathbf{u}_{i, j}=\hbar_{j}^{-1}\left[A_{j+1, i}\left(\mathbf{u}_{j+1, i}-\mathbf{u}_{j, i}\right)-A_{j, i}\left(\mathbf{u}_{j, i}-\mathbf{u}_{j-1, i}\right)\right]+\Lambda^{*} \mathbf{u}_{i, j},
$$

where

$$
\begin{gathered}
\Lambda^{*} \mathbf{u}_{i, j}=\left(\hbar_{i}^{*}\right)^{-1}\left[A_{j, i+1}^{*}\left(\mathbf{u}_{j, i+1}-\mathbf{u}_{j, i}\right)-A_{j, i}^{*}\left(\mathbf{u}_{j, i}-\mathbf{u}_{j, i-1}\right)\right], \\
A_{j, i}=\frac{\lambda_{j-0.5, i}}{h_{j}} g\left(-\beta_{j, i}\right), A_{j+1, i}=\frac{\lambda_{j+0.5, i}}{h_{j+1}} g\left(\beta_{j+1, i}\right), \\
A_{j, i}^{*}=\frac{\lambda_{j, i-0.5}}{h_{i}^{*}} g\left(-\beta_{j, i}^{*}\right), A_{j, i+1}=\frac{\lambda_{j, i+0.5}}{h_{i+1}^{*}} g\left(\beta_{j, i+1}^{*}\right), \\
\beta_{j, i}=\left(\lambda_{j-0.5, i}\right)^{-1} a_{j-0.5, i} h_{j}, \beta_{j, i}^{*}=\left(\lambda_{j, i-0.5}\right)^{-1} a_{j, i-0.5}^{*} h_{i}^{*}, \\
h_{i}^{*}=y_{i}-y_{i-1}, h_{i+1}^{*}=y_{i+1}-y_{i}, j=\overline{1, N-1}, i=\overline{1, N^{*}-1}, y_{N^{*}}=l^{*} .
\end{gathered}
$$

We again can estimate the truncation error in the space

$$
O\left(\max \left|h_{j+1}-h_{j}\right|+\max \left|h_{i+1}^{*}-h_{i}^{*}\right|\right),
$$

or $O\left(h^{2}+\left(h^{*}\right)^{2}\right)$ in the case of the uniform grid with steps $h, h^{*}$. The difference scheme with piece-wise constant functions $\lambda, a, a^{*}, \mathbf{f}^{*}$ is not exact.

\section{2D STEADY-STATE SHEAR FLOW}

The 2D stady state shear flow of viscous incompressible electrically-conducting liquid and magnetic induction field can be described by the system of two linear MHD equations (the Hunt type equations) [7]:

$$
\begin{aligned}
& \Delta u+H a\left[\cos \left(\alpha_{0}\right) \partial H / \partial x+\sin \left(\alpha_{0}\right) \partial H / \partial y\right]=\operatorname{Rev} \partial u / \partial y \\
& \Delta H+H a\left[\cos \left(\alpha_{0}\right) \partial u / \partial x+\sin \left(\alpha_{0}\right) \partial u / \partial y\right]=\operatorname{Re}_{m} v \partial H / \partial y
\end{aligned}
$$

where the functions $u, H$ are the corresponding unknown components of velocity and magnetic induction vectors, $R e, R e_{m}, H a$ are the Reynolds, magnetic Reynolds and Hartmann numbers, $\alpha_{0}$ is the angle between the directions of $x$ axes and external magnetic field, $v$ is the given function of $y$ components of velocity vectors. It follows from (12), (13) that the vectors $\mathbf{u}=(u, H), \mathbf{f}^{*}=(0,0)$, the matrix $\lambda=E$,

$$
a=-H a\left(\begin{array}{cc}
0 & \cos \left(\alpha_{0}\right) \\
\cos \left(\alpha_{0}\right) & 0
\end{array}\right), a^{*}=\left(\begin{array}{cc}
R e v & -H a \sin \left(\alpha_{0}\right) \\
-H a \sin \left(\alpha_{0}\right) & R e_{m} v
\end{array}\right) .
$$


Then the difference scheme ( $\sigma=0, \tau=\infty$ ) on the uniform grid can be written in the following form

$$
\Lambda \mathbf{u}_{j, i}=\gamma \delta_{x}^{2} \mathbf{u}_{j, i}+\gamma^{*} \delta_{y}^{2} \mathbf{u}_{j, i}-a \delta_{x} \mathbf{u}_{j, i}-a^{*} \delta_{y} \mathbf{u}_{j, i}=0,
$$

where

$$
\begin{gathered}
\gamma=\left(\begin{array}{cc}
g_{0} & 0 \\
0 & g_{0}
\end{array}\right), \quad \gamma^{*}=\left(\begin{array}{cc}
a_{1,1} & a_{1,2} \\
a_{1,2} & a_{2,2}
\end{array}\right), \\
a_{1,2}=H a \sin \left(\alpha_{0}\right)\left(g\left(\mu_{2}\right)-g\left(\mu_{1}\right)\right) /\left(\mu_{2}-\mu_{1}\right), \\
a_{1,1}=\left[g\left(\mu_{2}\right)\left(\mu_{2}+R e_{m} v\right)-g\left(\mu_{1}\right)\left(\mu_{1}+R e_{m} v\right)\right] /\left(\mu_{2}-\mu_{1}\right), \\
a_{2,2}=\left[g\left(\mu_{1}\right)\left(\mu_{2}+R e_{m} v\right)-g\left(\mu_{2}\right)\left(\mu_{1}+R e_{m} v\right)\right] /\left(\mu_{2}-\mu_{1}\right), \\
\mu_{1,2}=\mu_{ \pm}=-0.5\left[v\left(R e+R e_{m}\right) \pm \sqrt{d}\right], d=v^{2}\left(R e-R e_{m}\right)^{2}+4 H a^{2} \sin ^{2}\left(\alpha_{0}\right), \\
g\left(\mu_{k}\right)=0.5 h^{*} \mu_{k} \operatorname{coth}\left(0.5 h^{*} \mu_{k}\right),(k=1 ; 2), \\
g_{0}=0.5 H a \cos \left(\alpha_{0}\right) \operatorname{coth}\left(0.5 H a \cos \left(\alpha_{0}\right)\right),
\end{gathered}
$$

$\delta \mathbf{u}, \delta^{2} \mathbf{u}$ denote the central difference expressions for the first and second order. The difference equations (15) have been applied for large values of parameters $R e, R_{m}, H a[7]$.

\section{2-D HYDRODYNAMIC SYSTEM}

The subject of examination is the finite difference approximations of the flow equations describing 2-D incompressible flow in curvilinear orthogonal coordinates $\left(q_{1}, q_{2}, q_{3}\right)$ in the case when it is possible to introduce the stream function $\psi$ of the liquid. The planar flow is governed by the Navier-Stokes equation in the Crosso-Lamb form in terms of stream function $\psi, q_{3}$-components of vorticity $\omega_{3}$ and velocity $v_{3}$ (all derivatives along the coordinate $q_{3}$ are equal to zero). Elimating the pressure, we obtain the system of two equations for the functions $\omega_{3}$ and $v_{3}$ with the 'source' terms of the form $b v_{3}, d \omega_{3}$, where the functions $b, d$ can change their signs. This causes difficulties in the derivation of monotone difference schemes, since the maximum principle is not valid for such equations. Using the transformation $u=L_{3}^{-1} \omega_{3}, w=L_{3} v_{3}$ and taking into account the relation

$$
\partial\left(L_{1}^{-1} L_{2} \partial L_{3} / \partial q_{1}\right) / \partial q_{1}+\partial\left(L_{2}^{-1} L_{1} \partial L_{3} / \partial q_{2}\right) / \partial q_{2}=0
$$

the equations for $u, w$ do not contain source terms and therefore, are suitable for obtaining a monotone vector-difference scheme $(12,13)$ [8]. Here, $L_{1}, L_{2}, L_{3}$ are the Lames coefficients of the orthogonal-coordinates transformation. Let 
us note that in any orthogonal coordinate system, the relationship (16) is valid. The 1-D steady-state model of two equations of this system is in the form (1), where

$$
\lambda=\left(\begin{array}{cc}
b_{1} & 0 \\
0 & b_{2}
\end{array}\right), \quad a=\left(\begin{array}{cc}
a_{1} & c \\
0 & a_{2}
\end{array}\right)
$$

are the matrixes of the second order depending on variable $x, b_{1}>0, b_{2}>0$, $\mathbf{u}, \mathbf{f}$ are the vectors with two components $(u, \omega)$ and $\left(f_{1}, f_{2}\right)$.

For the finite-difference approximation of systems (1) on nonuniform grid the system can be written in the following local selfadjoint form (4) and the monotone vector-difference equations of 3 -point scheme can be represented in the form (6), where

$$
\mathbf{u}_{j}=\mathbf{u}\left(x_{j}\right), \mathbf{f}_{j}=\mathbf{f}\left(x_{j}\right), \mathbf{R}_{j}=\mathbf{f}_{j} / \hbar_{j} .
$$

By calculation the matrix-function $g(s)$ on the spectrum of the matrix $s=$ $\lambda^{-1} a h$ we get

$$
g(s)=\left(\begin{array}{cc}
g\left(k_{1}\right) & c b_{2}^{-1} g\left(k_{1}, k_{2}\right) h \\
0 & g\left(k_{2}\right)
\end{array}\right),
$$

where

$$
\begin{gathered}
k_{i}=a_{i} b_{i}^{-1} h(i=1,2), \quad g(k)=k /(\exp (k)-1), \\
g\left(k_{1}, k_{2}\right)=\left(g\left(k_{1}\right)-g\left(k_{2}\right)\right) /\left(k_{1}-k_{2}\right), \quad \lim _{k_{2} \rightarrow k_{1}} g\left(k_{1}, k_{2}\right)=g^{\prime}\left(k_{1}\right) .
\end{gathered}
$$

The presence of matrix- function $g(s)$ in the case of piece-wise constant functions of $b_{1}, b_{2}, a_{1}, a_{2}, c \quad(\mathbf{f}=\mathbf{0})$ ensures the exact discrete approximation of the boundary value problem for equation (1).

\section{2-D MHD SYSTEM}

The class of axially symmetric or planar 2-D flow of viscous incompressible electrically-conducting liquid can be described by the system of MHDequations in terms of six unknown functions in curvilinear orthogonal coordinates $\left(q_{1}, q_{2}, q_{3}\right)$ and in time $t$ : temperature $T$, stream functions of the liquid $\psi$ and the magnetic field $\kappa, q_{3}$-components of vorticity $\omega_{3}$, velocity $v_{3}$ and magnetic induction $B_{3}$ (all derivatives along the coordinate $q_{3}$ are equal to zero). Elimating the pressure, we obtain three equations for the functions $v_{3}, B_{3}, \omega_{3}$ with the 'source' terms of the form $b v_{3}, c B_{3}, d \omega_{3}$, where $b, c, d$ are the alternating sign functions. This makes the development of the monotone difference scheme more complicated, since the maximum principle is not valid. Using the transformation

$$
u=L_{3}^{-1} \omega_{3}, w=L_{3} v_{3}, H=L_{3}^{-1} B_{3}
$$


and the relationship (16), we obtain the equations for $u, w, H$ which do not contain source terms, therefore, they are suitable for obtaining monotone vector-difference schemes $(12,13)$ [8].

The 1-D steady-state model system is in the form (1), where

$$
\lambda=\left(\begin{array}{ccc}
b_{1} & 0 & 0 \\
0 & b_{2} & 0 \\
0 & 0 & b_{3}
\end{array}\right), \quad a=\left(\begin{array}{ccc}
a_{1} & c & d \\
0 & a_{2} & 0 \\
0 & 0 & a_{3}
\end{array}\right)
$$

are the matrixes of the third order depending on variable $x, b_{1}>0, b_{2}>$ $0, b_{3}>0, \mathbf{u}, \mathbf{f}$-the vectors with three components $(u, w, H)$ and $\left(f_{1}, f_{2}, f_{3}\right)$.

For the finite- difference approximation of systems (1) on nonuniform grid with grid points $x_{j}$ the system can be written in the following local selfadjoint form (4) and the monotone vector-difference equations of 3-point scheme can be represented in the form (6). By calculation the matrix-function $g(s)$ on the spectrum of the matrix $s=\lambda^{-1} a h$, we get

$$
g(s)=\left(\begin{array}{ccc}
g\left(k_{1}\right) & c b_{2}^{-1} g\left(k_{1}, k_{2}\right) h & d b_{3}^{-1} g\left(k_{1}, k_{3}\right) h \\
0 & g\left(k_{2}\right) & 0 \\
0 & 0 & g\left(k_{3}\right)
\end{array}\right)
$$

where

$$
\begin{gathered}
k_{i}=a_{i} b_{i}^{-1} h(i=1,2,3), \quad g(k)=k /(\exp (k)-1), \\
g(\tilde{k}, k)=(g(\tilde{k})-g(k)) /(\tilde{k}-k), \quad \lim _{\tilde{k} \rightarrow k} g(\tilde{k}, k)=g^{\prime}(k) .
\end{gathered}
$$

In the case of piece-wise constant functions $b_{1}, b_{2}, b_{3}, a_{1}, a_{2}, a_{3}, c, d,(\mathbf{f}=\mathbf{0})$ the presence of the matrix- function $g(s)$ ensures the exact discrete approximation of boundary value problem for equation (1).

\section{SOME APPLICATIONS}

The main result of numerical modelling in continuum mechanics is the development of effective special calculation methods for obtaining electromagnetic, hydrodynamic and temperature fields with various boundary layers [7]. Special finite-difference methods have been applied for the simulation of liquid transport in electrolytic cell for aluminium production [9], in multilayered fleece for describing the wetting-drying process [10],[11], for mathematical modelling of the glass fibre material production[12],for the solutions of filtration and heat transfer problems in multilayer domains[13],[14]. 
The exact finite-difference scheme for vector boundary-value problems 123

\section{REFERENCES}

[1] E.P.Doolan, J.J.H.Miller, W.H.A.Schilders. Uniform numerical methods for problems with initial and boundary layers, Dublin, 1980.

[2] A.M.Ilhyn. The difference scheme for differential equation with small parameters at second order derivatives. Mathematical note, 6, No.2(1969), 234-248 (in Russian).

[3] H.Kalis. Special finite-difference approximations for numerical solution of some linear and non linear differential equations. Proc. of the 2-nd conference "Numerical modelling in continuum mechanics" in Prague 22-25 August, 1994, Prague 1995, 133-138.

[4] J.W.Thomas.Numerical partial differential equations. Finite difference methods. Springer-Verlag, New-York,Inc., 1995.

[5] P.Lancaster. Theory of matrices. Acad. Press, New-York-London, 1969.

[6] H.Kalis.Numerical integration by finite-difference methods for some problems of linear and nonlinear systems of differential equations. Acta Universitatis Latviensis, Riga, 592(1994), 91-104.

[7] H.Kalis. Ausarbeitung und Anwendung der spezialen numerischen Methoden zu Losung der Probleme der mathematischen Physik,Hydrodinamik und Magnetohydrodynamik, Riga, Acta Universitatis Latviensis, 588(1993), 175-206.

[8] H.Kalis. Special finite-difference approximations of flows equations in term of stream function, vorticity and velocity components for viscous incompressible liquid in curvilinear orthogonal coordinates. Comment.Math. Univ.Carolinae 34), No 1(1993), 165174.

[9] A.Buikis,H.Kalis. The mathematical simulation of an electrolytic cell for aluminium production. Proc. of 8-th int. conf. of ECMI in Kaiserslautern 6-10 Sept., 1994, Berichte der Arbeitsgruppe Technomathematik, Nr95-150, 1995.

[10] A.Zemitis,H.Kalis. About the mathematical simulations of the wetting-drying process. Latvian journal of physics and technical sciences, No 6(1995), 37-44.

[11] H.Kalis. The effective finite-difference methods for mathematical modelling of the liquid transport in fleece. Proc. of 3-rd int.conf. "Numerical modelling in continium mehanics", part 2, Praque, 1997, 314-320.

[12] J.Cepitis,H.Kalis. A certain mathematical model of the glass fibre material production. Proc. of 9-th int. conf. ECMI-96, Copenhagen, 1997, "Progress in Industrial Mathematics at ECMI-96", B.G.Teubner, Stuttgart, 1997, 166-173.

[13] H.Kalis. Effective finite-difference methods for the solutions of filtration problem in multilayer domains. Proc. of 2-nd int. conf. "Mathematical modelling and complex analysis", Vilnius, 1997, 84-91.

[14] H.Kalis.Some heat transfer problems with convection in multilayer media. Proc. of 2-nd int. conf. "Finite difference methods: theory and applications", Minsk-Belarus, vol.2, $1998,50-55$. 